\title{
Bücher im Frühling
}

\author{
Erhard Taverna
}

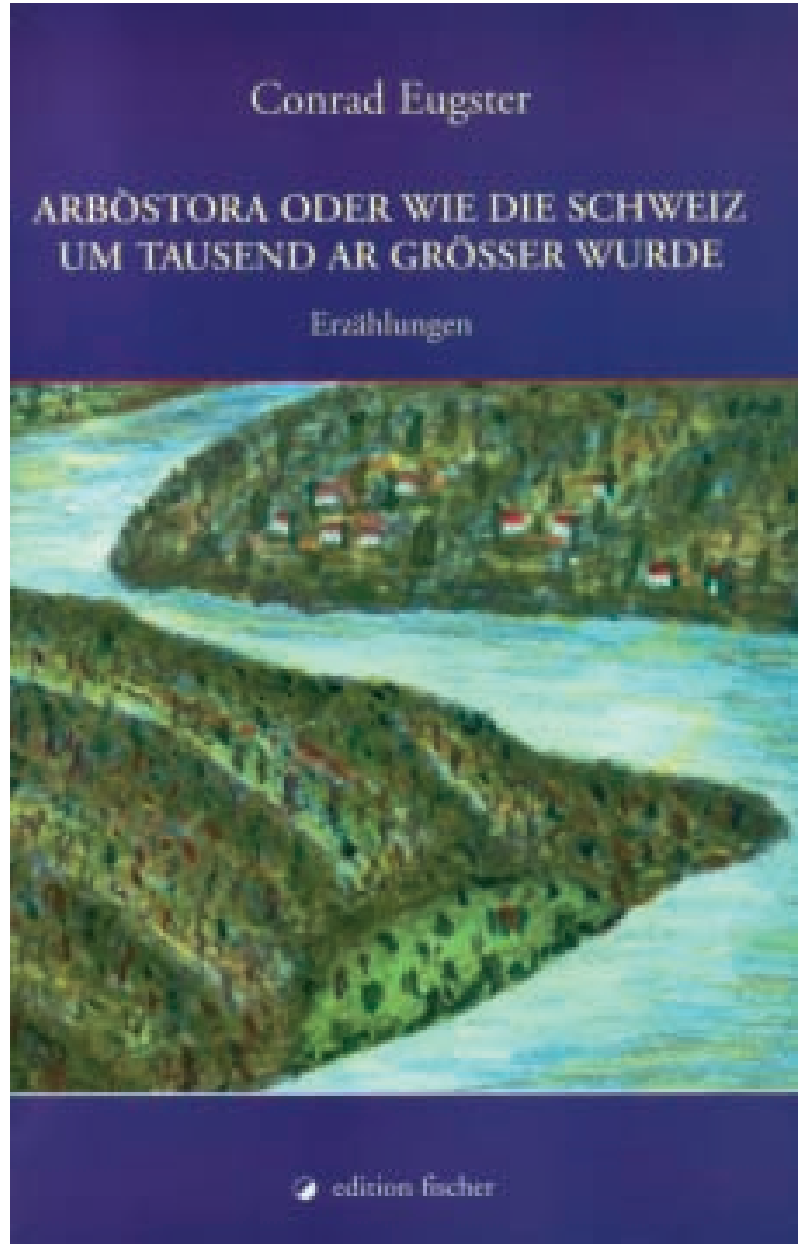

\section{Arbòstora oder wie die Schweiz um tausend Ar grösser wurde}

Der in St. Gallen praktizierende Internist Conrad Eugster hat sechs Erzählungen veröffentlicht. Wie viele Mediziner seines Jahrganges (1953) verdankt er dem Militärdienst die Entdeckung der Tessiner Landschaft, deren Besonderheiten er uns auf zwei Entdeckungsreisen vorstellt. In der Titelgeschichte begleiten wir den Erzähler auf einer langen Wanderung über die steilen Kehren hinauf zur Kirche von Vico Morcote und durch Kastanienhaine und Laubwälder in die Umgebung von Morcote bis an den seenahen Südhang des Monte Arbòstora. Die wortreich verschachtelten Haupt- und Nebensätze erzwingen einen Leserhythmus, der sich präzise dem Schritttempo anpasst. Nicht nur, dass dem Gehetzten bald der Atem ausginge, er würde, schlimmer noch, einen Bergfrühling verpassen, dessen Duft und Glanz der Autor meisterhaft aus seiner Sprachfülle zum Leben erweckt. Eugster ist ein Baumfreund und Blumenliebhaber, der mit dem Blau aus der Farbenpalette südlicher Blumenwiesen immer wieder seine Sehnsuchtsblüte Jasione ausmalt: «wobei die aufgehenden Blütenköpfe die hellblaue Tönung des Morgens, die offenen Blumen die satte Farbe des Mittags und die verblühenden, welk gewordenen Schöpfe das tiefe Dunkel des vorgerückten Abendhimmels wiedergaben.» Die lyrische Reise kippt ins Phantastische mit der unerwarteten Entdeckung eines, den schweizerischen Landestopographen entgangenen, Naturgartens, einer Entdeckung allerdings, die den Behörden nur Ärger bereitet, da auch ein Paradies, durch die nachzutragende Kartierung, Kosten verursacht. Den Tag und das Leben begleitet die blaue Jasione, vom ersten Eindringen in das verbotene Gelände bis zum Rentnereinzug in das inzwischen parzellierte Glück. In «Klingsor» begegnet der Autor im Ristorante della Posta seinem verehrten Herrmann Hesse, der sich dort, obwohl längst verstorben, mit seiner Gefährtin Ninon aus der gleichen Speisekarte verpflegt. Dämonisch und nahtlos kippt der Alltag auf der «Piazza Navone», an einem hellen Kongressnachmittag in Rom, in eine surreale Parallelwelt, wo die Kunstschätze des Palazzo Pamphili zur abgründigen Falle geraten. Conrad Eugster erweist sich mit diesem Band als scharfer Beobachter, dem mit seinem üppigen Sprachstil und seinem Sinn für die unheimlichen Randzonen des Lebens eine unterhaltende Lektüre gelungen ist. Wer mehr über den Autor erfahren will, findet weitere Angaben in seiner Homepage www.conradeugster.ch.

- Eugster C. Arbòstora oder wie die Schweiz um tausend Ar grösser wurde. Erzählungen. Frankfurt/Main: R. G. Fischer Verlag; 2004. 176 Seiten.

\section{Schaffhauser Spitalgeschichte}

Arthur Uehlinger, medizinischer Chefarzt von 1968 bis 1993 am Schaffhauser Kantonsspital, hat im Auftrag der Chefärztekonferenz die Entwicklung vom städtischen Krankenhaus von 1848 bis zum spezialisierten kantonalen Zen- 


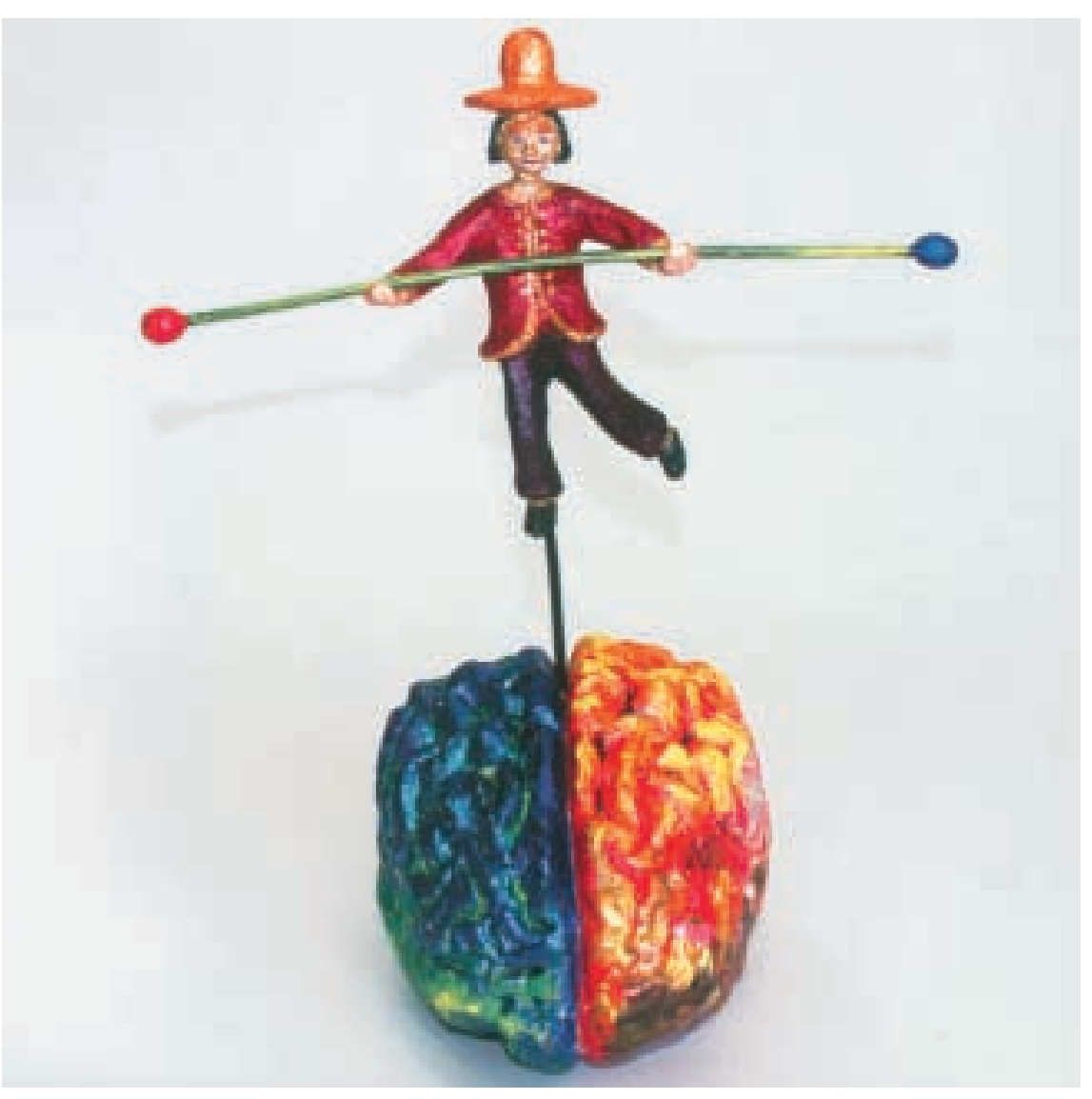

Masha Dimitri: Il fumambolo tra le emisfere.

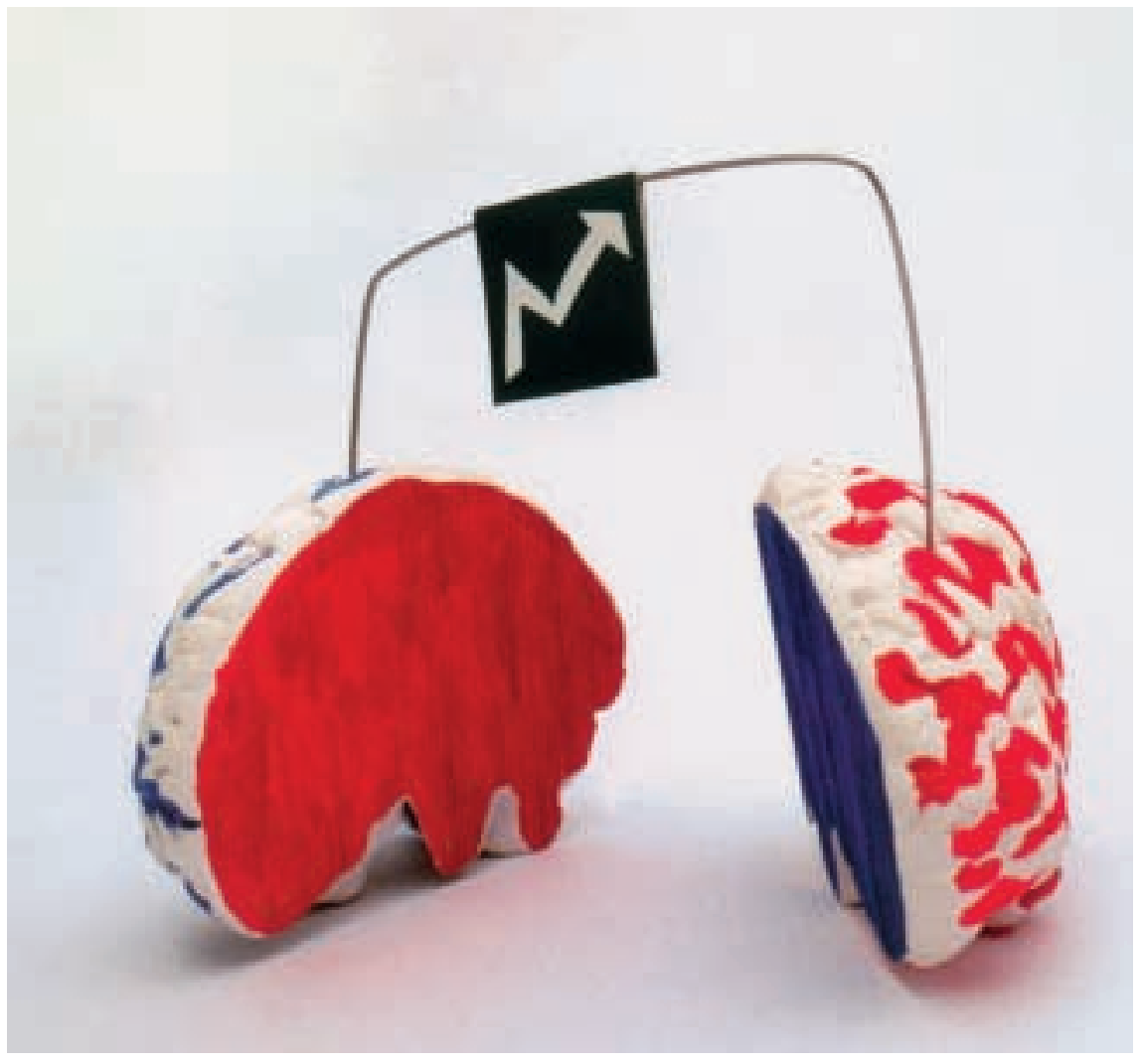

Frédéric Bolli: Hochspannung. trum 2002 nachgezeichnet. Das drucktechnisch aufwendig gestaltete Buch ist übersichtlich gegliedert und mit zahlreichen Tabellen, Bildern, Literaturangaben und Querverweisen bestückt. Die Chronik schildert den typischen Weg einer schweizerischen Klinik als Resultat des medizinischen und gesellschaftlichen Wandels. Zahlreiche Dokumente parlamentarischer Debatten und behördlicher Beschlüsse illustrieren die demokratisch-öffentlich geführten Auseinandersetzungen. Stadtentwicklung und Grenznähe prägen die Spitalgeschichte genauso wie die lokale Politik, persönliche Vorlieben und Animositäten, Anekdoten, Finanzen und wirtschaftliche Unternehmen. Aufbruch und Stagnation wechseln mit jeder Generation, die medizinischen und politischen Akteure kommen ausführlich zu Wort. Der Band schliesst mit einem als «Perspektiven» überschriebenen Kapitel, das die aktuellen Probleme auflistet: Ziele der Medizin, Fortschrittsfalle, begrenzte Ressourcen und die bevorstehende Volksabstimmung zum neuen Entwurf eines Spitalgesetzes, dessen Chancen und Risiken schwierig abzuschätzen sind. Für alle, die einen persönlichen Bezug zum Schaffhauser Kantonsspital haben, tut sich hier eine Fundgrube auf. In unserem föderalen Staatswesen gibt oder gäbe es 25 weitere, ähnliche Spitalgeschichten zu lesen. Inhaltlich gleichen sie sich alle, formal sind viele Ansätze möglich. Das Einmannwerk besticht durch seine medizinzentrierte Geschlossenheit, dafür sind die Pflegeberufe, die Patienten und die frei praktizierenden Ärzte weniger zu Wort gekommen.

- Uehlinger A. Schaffhauser Spitalgeschichte 1848-2002. Jubiläumsschrift. Bezugsquelle: Sekretariat Verwaltungsdirektion, Kantonsspital, 8208 Schaffhausen, E-Mail: doris.staeheli @kssh.ch.

\section{Brain Works}

Eine ganz andere Jubiläumsidee hatte die Memory Klinik der Psychiatrischen Klinik Münsterlingen. Aus der Initiative der Mitarbeiterinnen und Mitarbeiter entstand die Aktion «Brain Works». Der Silikongussform entsprangen gipserne Gehirnrohlinge, die an 100 ausgewählte Personen aus allen Berufs- und Lebensbereichen verschickt wurden. Jedes Gehirn wurde dafür in Einzelarbeit gefertigt, nachgearbeitet, durch das Spitalteam verpackt und dem Adressaten zur freien Gestaltung übergeben. Bis zum September 2003 wurden 93 Objekte künstlerisch bearbeitet wieder zurückgeschickt. Als nächstes entstand nach den Ideen des Kurators vom Kunstmuseum 
des Kantons Thurgau in Ittingen eine Ausstellung im Foyer des Gemeinschaftszentrums. Vernissage und Jubiläumsfest gerieten zum erfolgreichen Gesamtkunstwerk, aus dessen abschliessender Auktion eine namhafte Spende in die Kasse der Alzheimervereinigung Thurgau überwiesen wurde.

Das Buch zur A(u)ktion mit dem Titel «Brain Works» mit Photos von Ursula Huber schildert die Umsetzung der originellen Projektidee und besticht durch die Bilder und Begleittexte zu den bunten Exponaten. Erstaunliches ist hier zusammengekommen, ein Bilderbuch hirnrissiger Entdeckungsreisen, ernst, kurios, humorvoll und traurig, immer einfallsreich und anregend, verblüffend, belehrend und mit jedem Beispiel zur Nachahmung animierend. Das ansprechend gestaltete Werk regt zu eigenen Aktionen an und kann allen kreativen Akteuren im Gesundheitswesen, einschliesslich Verwaltungsmenschen, Politikern, Künstlern, Patienten und Angehörigen empfohlen werden.

- Brain Works. Das Buch zur Kunst A(u)ktion. 1. Auflage. 2003 Verlag: Memory Klinik, Psychiatrische Dienste Spital Thurgau AG, Postfach 154, 8596 Münsterlingen, www. stgag.ch, Druck: Huber \& Co., Frauenfeld, www. huber.ch.

\title{
Evidence-based und/oder Patient-based?
}

\author{
W. Zimmerli
}

Korrespondenz: Dr. med. Wolf Zimmerli Bahnhofstrasse 9 CH-3672 Oberdiessbach
Vor 40 Jahren besuchte ich meinen ersten Chirurgenkongress, dem ich als Assistent im Kantonsspital nur unter der Bedingung beiwohnen durfte, nachher einen zusammenfassenden Vortrag über die wesentlichen Erkenntnisse zu halten. Nebenbei kam ein solcher Besuch nur auf eigene Kosten und nur in Frage, wenn kein Dienst anfiel. Ich erinnere mich noch an die etwa vier guten Freien Mitteilungen und an die etwa fünf eindrücklichen Statements der grossen Chefs. Die ersteren mit eindrücklichen schönen Dias, die man lesen konnte, die zweiten als Excathedra-Vorlesungen. Alle anderen Vorträge waren nicht erwähnenswert, weil sie entweder «ein Fall von» ohne Signifikanz oder langweilig, kaum verständlich, mit schlechten oder keinen Dias und voll abgeschriebener Platitüden waren. Prof. Nissen, damals Präsident der Chirurgengesellschaft, hat dann mit seinem bekannt scharfen Verstand und seiner bisweilen recht bissigen Zunge die schlechten Referenten entweder durch Stellen der kritischen Fragen oder durch Blossstellen der Fehler richtiggehend in den Boden gestampft. Andere Vorsitzende haben den schlechten Referaten eher freundlich andere Alternativen gegenübergestellt. Sie waren nicht so direkt, wie einst Prof. Bleuler, Psychiater im Burghölzli, als er einem praktizierenden Studenten sagte: «Sehr richtig, Herr Kollege, aber es ist natürlich komplett falsch, was Sie da gesagt haben», aber im gleichen Sinn mit ein wenig Zuckerbrot vor der kräftigen Peitsche.
Die Kongresse und auch die internen Weiterbildungen und die Symposien wurden dann Jahr für Jahr besser und ein Referat an einem solchen Kongress wurde nur noch angenommen, wenn nach einer sauberen Vorstellung der Problematik und zum Beispiel einer neuen Operationstechnik eine wahrheitsgemässe und sauber durchgeführte Nachkontrolle der Fälle mit einer rein gefühlsmässig (noch nicht nach Signifikanz) genügenden Anzahl der Fälle präsentiert wurde.

Eine andere Art der Korrektur von schlechten wissenschaftlichen Sitten erlebte ich in Frankreich an den Tagungen des GEM (Groupe d'Etude de la Main), wo nur die jungen Assistenten oder Oberärzte die Vorträge und Präsentationen zu machen hatten. In der ersten Reihe sassen die Patrons und zerzausten mit romanischer Eloquenz und mit dem Fundus des Wissens, der Erfahrung und der Literaturkenntnis jeden unsauberen Vortrag, natürlich auch manchmal zu Unrecht.

Die Frage: «Würden Sie diese Operation oder diese Behandlungsart bei Ihrer Frau oder Ihrem Vater anwenden?» war häufig zu hören.

Es drangen dann zunehmend, von Amerika her kommend, drei wichtige Dinge in unser vor allem germanisch ererbtes «Nur gute Resultate»Denken.

- Komplikationenkonferenz: Einmal wöchentlich oder mindestens einmal pro Monat wurden alle Komplikationen der Klinik von den Assistenten vorgetragen und gemeinsam analysiert. 
- Doppelt-Blind-Studien oder prospektive Studien: Eine retrospektive Fallkontrolle und eine darauf basierende Studie, vor allem, wenn sie der Operateur selber durchgeführt hat, wurde nicht mehr als aussagekräftig taxiert und aus jeder Betrachtung ausgeschlossen.

- Evidence-based Medicine (EBM) oder deutsch evidenzbasierte Medizin: Es werden nur noch Massnahmen am Patienten geduldet und wohl auch in der Zukunft nur noch von den Krankenversicherern bezahlt, die aufgrund von Studien, und zwar eben prospektiven und genau auf Pferdefüsse durchleuchteten Studien, als statistisch signifikant gekürt worden sind.

Vor einigen Tagen besuchte ich ein Symposium, das aus brillanten Vorträgen von etwa zehn Superexperten über ein kleines Teilthema der Chirurgie abgehalten wurde. Ich ging tief beeindruckt von den durchwegs evidenzbasierten Vorträgen nach Hause. Sie wurden alle mit wunderschönen PowerPoint-Präsentationen vorgetragen, wo Texte von rechts nach der Mitte, von der Mitte nach aussen, von oben nach unten und auch noch animiert sich in rasender Abfolge, sich der Filmfrequenz von 18 Bildern pro Sekunde annähernd, ablösten.

Anderntags auf dem morgendlichen Hundespaziergang drehte sich die erlebte Brillanz der Fortbildung wie ein Karussell in meinen Hirnwindungen herum. Zwei Denkgegner verfolgten sich mit dem Zweihänder bewaffnet in den Gyri und suchten den Zweikampf. Das Duell spielte sich etwa so ab:

Der erste war begeistert über die Entwicklung des wissenschaftlichen Denkens, Publizierens, Vortragens und Arbeitens in den letzten 40 Jahren. Er lobte die signifikant besser gewordenen Resultate der Chirurgie, die gewachsene Selbstkritik der früher so narzisstischen Chirurgen, die Spezialisierung, die Konzentration von schwierigen Fällen auf wenige Zentren, wo der nötige Case-Load erreicht wird, die Durchführung noch nicht etablierter Verfahren ausschliesslich im Rahmen von Multizenterstudien und vor allem das Pochen auf die Evidence-Based Medicine als allein selig machendes Credo.

Der zweite bemängelte, dass am erwähnten Symposium auf jedem neuen Dia - darf man bei PowerPoint noch so sagen? - neben der gross aufgemachten Aussage ganz klein die fünf bis zehn evidenzbasierten Arbeiten vor dem Auge vorbeirauschten. Der Zuhörer hatte genau gleich wie beim Ex-cathedra-Vortrag vor 40 Jahren daran zu glauben, dass der Referent die richtigen Arbeiten aus dem Internet herausgefiltert hat. Dieser zweite Robin Hood hob dann den Zweihänder zum entscheidenden Schlag, liess ihn dann aber wieder sinken und sagte bescheiden: Sehr richtig, Herr Kollege, aber es ist natürlich grundfalsch, was Sie da gesagt haben. Vor 40 Jahren und auch heute noch haben wir das zu glauben, was uns die Experten sagen. Deshalb sind für uns Chirurgen nur drei Dinge wichtig:

- die Kenntnis unseres Berufes, mit Anatomie, Literatur, manueller Geschicklichkeit, Erfahrung und der Fähigkeit, schnell den richtigen Entscheid zu treffen;

- Selbstkritik und Kennen der eigenen Grenzen; - als wichtigstes: Der Patient, der Leidende, der nicht eine statistische Zahl, sondern etwas Einzigartiges, in seiner ganzen Persönlichkeit Ernstzunehmendes ist, muss unsere Gedanken leiten. Das Eindringen in die Seele, in die körperliche Befindlichkeit, in die geheimen Ängste und Wünsche dieses Menschen muss beim Entscheid, was wir ihm empfehlen, und wie wir ihn operieren oder nicht operieren, leiten, mehr als die statistisch signifikanten, evidenzbasierten und prospektiven Studien.

Nach diesem nicht zu einem eindeutigen Sieg gelangten Duell bleiben für mich Fragen: Was ist nun wichtiger? Die EBM oder der Patient? Wahrscheinlich beides. Aber stimmen die Relationen noch? 